\title{
The pulse-phase-resolved spectroscopy of the Crab pulsar with SPI/INTEGRAL
}

\author{
S. Molkov* \\ Centre d'Etude Spatiale des Rayonnements \\ E-mail: molkov@cesr.fr \\ E. Jourdain, J. P. Roques and L. Borgonovo \\ Centre d'Etude Spatiale des Rayonnements
}

\begin{abstract}
We investigated spectral properties of the Crab nebular emission in the pulsar phase plane in the $0.02-1.2 \mathrm{Mev}$ energy band with the SPI telescope. After applying a barycentre correction procedure we folded publicly available SPI/INTEGRAL data of $\sim 3.4$ Ms Crab observations with radio monthly ephemeris (see [1]). We found that the shape of Crab phase histograms and absolute phases are very stable. In this work we focus attention on the spectral study of "Total pulsed" and "Unpulsed" emission from the Crab nebula. We define "Unpulsed" emission as an appropriately rescaled "Off pulse" phase component ("Unpulsed"="Off pulse"/(0.86-0.5)). "Total pulsed" is the total Crab nebular emission minus "Unpulsed". We assumed that pulsating emission is negligible for the "Off pulse" phase component. Both "Pulsed" and "Unpulsed" mean spectra could be well fitted with a broken powerlaw model. We showed that the spectral shape and flux of both the components are very stable in time. Moreover neither "Pulsed" nor "Unpulsed" mean spectra do not reveal emission line features. The $3 \sigma$ upper limits on possible $440 \mathrm{keV}$ red shifted positron annihilation line in "Pulsed" and "Unpulsed" emission are $3 \times 10^{-4}$ and $6 \times 10^{-4}$ $\mathrm{ph} / \mathrm{s} / \mathrm{cm}^{2}$, respectively.
\end{abstract}

7th INTEGRAL Workshop

September 8-11 2008

Copenhagen, Denmark

${ }^{*}$ Speaker. 


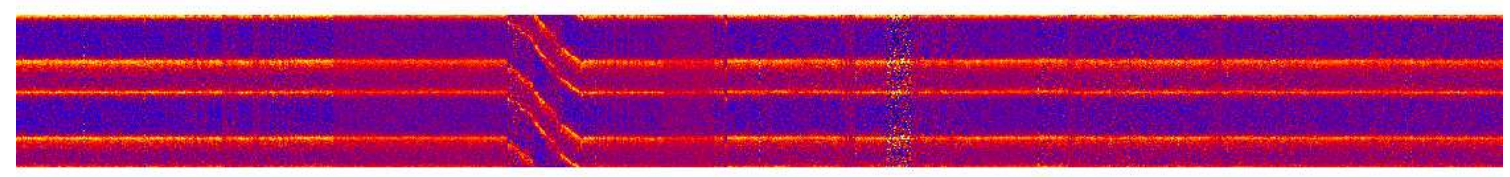

\section{Observations and data reduction}

In our analysis we have used publically available data of all observations where the source was in the FOV of the SPI telescope ( $<15 \mathrm{deg}$ ). The total exposure of selected data is approximately 3.4 Ms. There are two main types of SPI events: single events (registered by only one detector) and multiple events (with scattered photons detected by two and more detectors). We used only single events. For the conversion of the on-board time to Universal time and for the barycentric correction we used routines from the standard Off-line Science Analysis software package version 7.0 developed at the INTEGRAL Science Data Centre [2]. For other types of the SPI data analysis we used the calibration files and software developed at the Centre d'Etude Spatiale des Rayonnements.

To check our SPI data set for the presence of "glitches" from the Crab pulsar or some instrumental artifacts (e.g. inaccuracy in the on-board to Universal time conversion procedure) we folded separately each SCW (Science Window or pointing, a natural piece of INTEGRAL data - a pointing observation with exposure $\sim 2-5 \mathrm{ks}$, in our case there were $1356 \mathrm{SCWs}$ ) with radio monthly ephemeris (see [1]) in the broad 20-100 keV energy band. The result of the folding with a phase resolution 0.01 is presented in the header image. The shape of Crab phase histograms and absolute phases are very stable (two peaks of the pulse are good tracers) excluding one time interval with a significant phase drifting. This drift is the result of a large glitch that took place in Crab on Sept. 6, 2004. After taking it into account we did not find any other deviations from the radio ephemeris.

To show the change in the pulse profile with energy we divided the phase period in 50 equal intervals. In Fig. 1 we show Crab phase histograms in 10 energy bands (indicated on the top of each frame) in absolute phase with the phase resolution 0.02. Phase intervals from the Table 1 are highlighted with colours. The evolution of the pulse profile with energy is obvious. In the softer energy bands the first peak is more powerful than the second one while at the higher energies the

\begin{tabular}{|ll|c|}
\hline \multicolumn{1}{|c|}{ Component } & & Phase interval \\
\hline Leading Wing 1 & LW1 & $0.86-0.92$ \\
Peak 1 & P1 & $0.92-1.02$ \\
Trailing Wing 1 & TW1 & $1.02-1.12$ \\
Bridge & Bridge & $1.12-1.23$ \\
Leading Wing 2 & LW2 & $1.23-1.30$ \\
Peak 2 & P2 & $1.30-1.41$ \\
Trailing Wing 2 & TW2 & $1.41-1.50$ \\
Off pulse & OP & $0.50-0.86$ \\
Total pulse & TP & $0.86-1.50$ \\
\hline
\end{tabular}

Table 1: Definitions of the phase components 


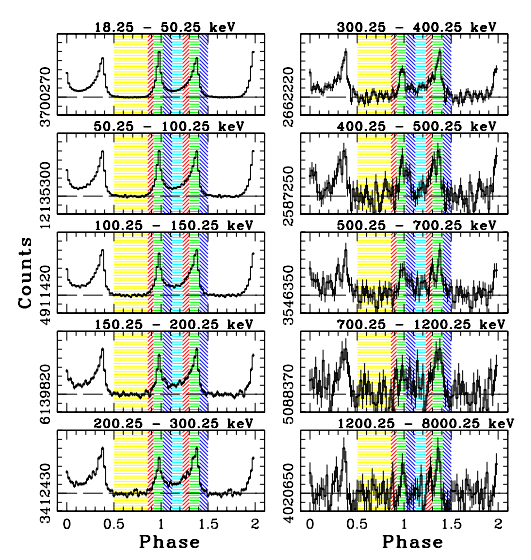

Figure 1: Crab phase histograms in 10 energy bands (shown at the top of each frame). The phase is absolute, its resolution is 0.02 . Phase intervals from Table 1 are highlighted with colours.
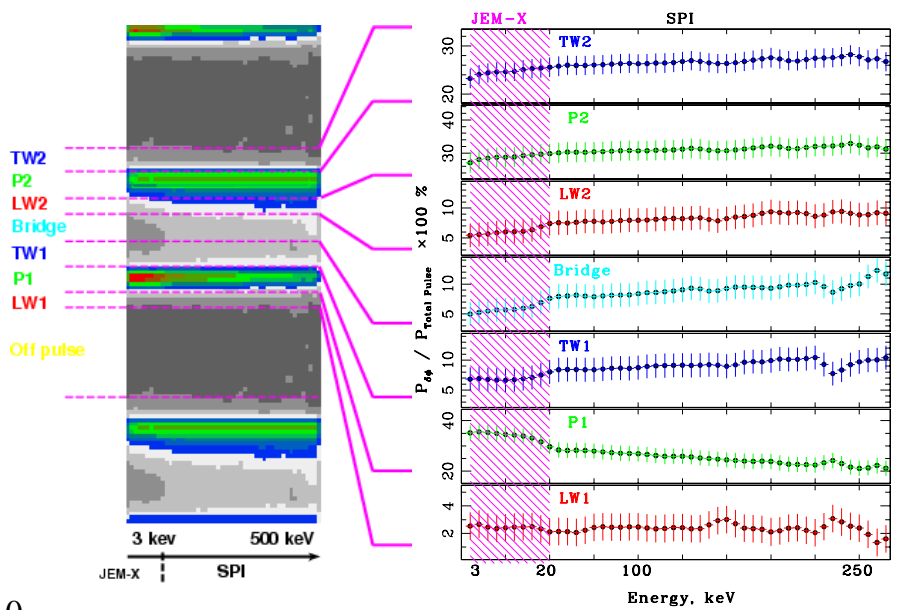

Figure 2: Evolution of the "Total Pulsed" component morphology depending on energy (left). Relative fraction of the pulsed components (listed in the table) to the "Total Pulsed" emission (right).

second peak becomes more prominent (see also the Fig.2). Such changes in the morphology have been observed before (see e.g. [2]). The figure demonstrates also that we can detect pulsating signal in the whole SPI energy range.

For detailed investigation of the morphology of the "Total Pulsed component" depending on energy in details we rebinned the phase intervals into several main groups representing different phase components (Table 1; the definition of these components was introduced in previous works, see e.g. [3]) and split the SPI energy band into 50 energy bins. The result is presented on the left part of the Fig.2. On the right part of the Fig.2 we shown relative fraction of the pulsed components (listed in the table) to the total "Pulsed emission". For completeness we added ten energy bins in the 3-20 keV energy band from JEM-X. We see that the main contribution to the change in the distribution of power among the phase components comes from the Peak 1 which relative fraction to the total "Pulsed emission" is continuously decreasing from $\sim 40 \%$ in the soft energy band to $\sim 20 \%$ in the hard one.

\section{Results}

In this work we focus attention on the spectral study of the "Total pulsed" and "Unpulsed" emission from the Crab nebula (see [4] for spectral analysis of the "Total" emission from the Crab nebula). We define the "Unpulsed" emission as an appropriately rescaled the "Off pulse" phase component ("Unpulsed"="Off pulse" $/(0.86-0.5))$. The "Total pulsed" is the total Crab nebular emission minus the "Unpulsed". We assumed that the pulsating emission is negligible for "Off pulse" phase component.

First of all we checked how stable are spectra of the "Total pulsed" and "Unpulsed" components with time. For this we fitted separately spectra of both the components for each revolution of our data set with the broken powerlaw model $\left(E_{0}=100 \mathrm{keV}\right.$, fixed). Records of the "Total 

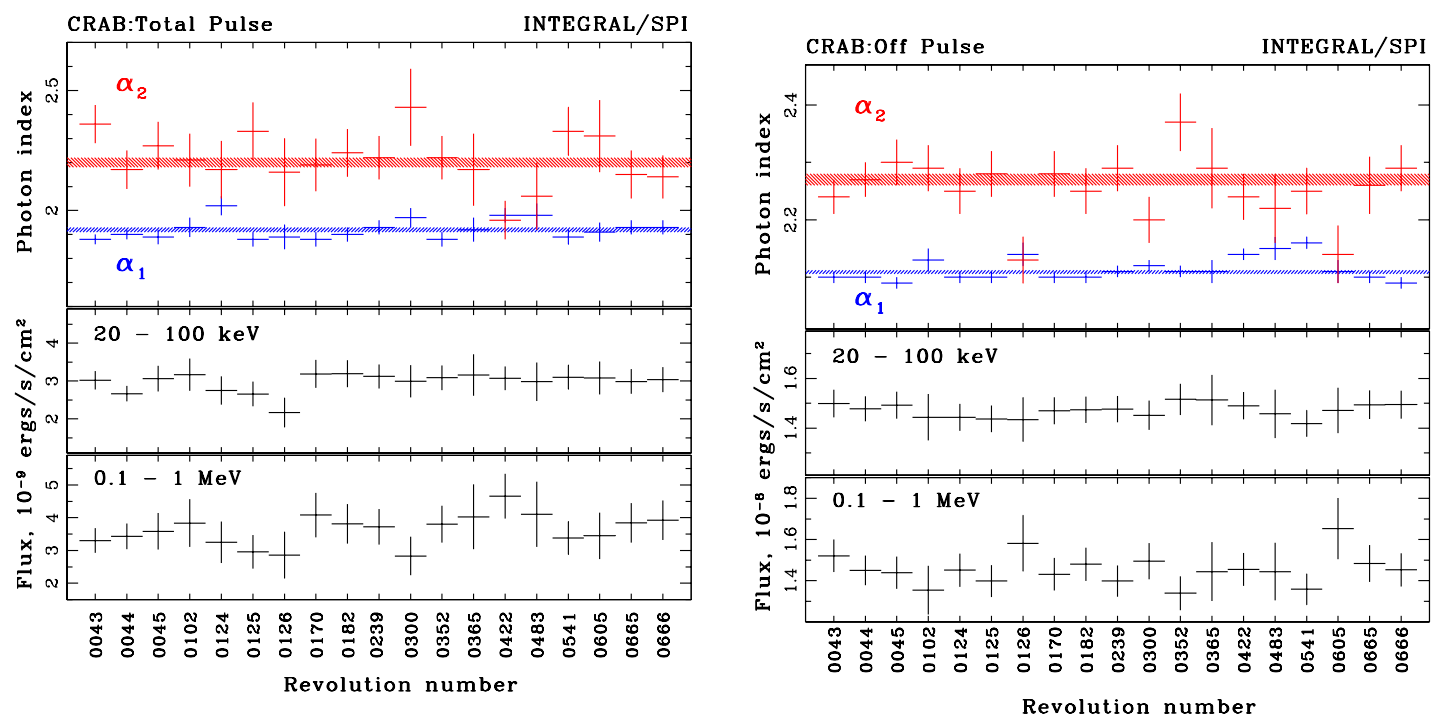

Figure 3: Records of the "Total pulsed" (left) and "Unpulsed" (right) model fluxes from the Crab nebula measured at different revolutions in two broad energy bands.

pulsed" and "Unpulsed" model fluxes in two broad energy bands as well as the two photon indexes of the the broken powerlaw model are shown in the Fig.3. The $\pm 1 \sigma$ uncertainty intervals assuming the constant spectral index parameters are indicated by the shaded regions. We found that there is no evidence of variability of the fluxes neither "Total pulsed" nor "Unpulsed" emission. Spectral shapes of both the components are stable in time (note, that significant flux and spectral variations of the CRAB emission were observed with BATSE, see [5]).

As the spectral parameters of Crab do not depend on time we can increase statistics by means summing up all available data. In Fig.4 we presented the mean "Total pulsed" and "Unpulsed" spectra with the best fits and fit's parameters of the Broken Powerlaw model. On the bottom panel we show evolution of the "Unpulsed"/"Total pulsed" ratio with energy. The spectrum of the "Total pulsed" emission is essentially harder than the spectrum of the "Unpulsed" one up to $100 \mathrm{keV}$ and both spectra have approximately the same slope above this energy.

\section{Conclusions}

We can summarise the results of this job as follows:

1. Both the "Pulsed" and "Unpulsed" mean spectra could be well fitted with a broken powerlaw model.

2. We found that the spectral shape and flux of both the components are very stable with time.

3. Neither "Pulsed" nor "Unpulsed" mean spectra do not demonstrate emission line features. The $3 \sigma$ upper limits on flux from the possible $440 \mathrm{keV}$ red shifted positron annihilation line (see also [6], [7], [8]) are $3 \times 10^{-4}$ and $6 \times 10^{-4} \mathrm{ph} / \mathrm{s} / \mathrm{cm}^{2}$, in "Pulsed" and "Unpulsed" emission respectively. These upper limits are higher than the line intensity that was observed previously with other instruments $([6],[7])$. 


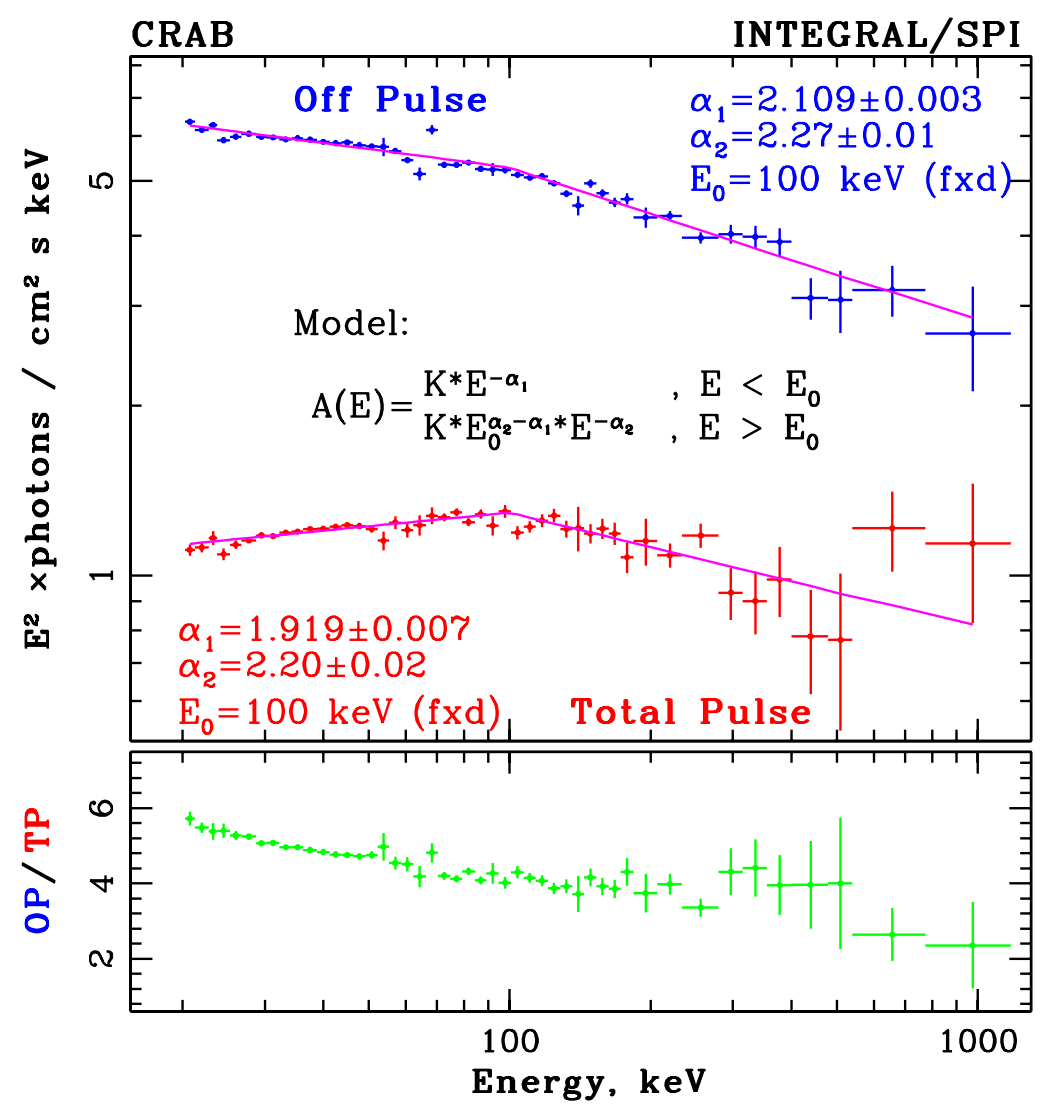

Figure 4: The mean "Total pulsed" and "Unpulsed" spectra with the best fits

\section{Summary}

The SPI telescope has a stable instrumental background, a good response matrix and high enough flux sensitivity to make fine phase resolved spectroscopy of the emission from the pulsar from $\sim 25 \mathrm{keV}$ up to a few MeV. In future we are planning to use SPI data for the similar analysis of high energy emission from other pulsars.

\section{References}

[1] Lyne et al. 2008, IJodrell Bank Crab pulsar timing results;

[2] Courvoisier et al. 2003, A\&A, 411, L53

[3] Kuiper et al., A\&A 378, 918-935, 2001

[4] Jourdain and Roques 2008, in proceedings of the 7th INTEGRAL Workshop,'PoSS(Integral08)1433'

[5] Ling et al., Astroph.J., 598, 334-348, 2003

[6] Mineo et al., A\&A 450, 617-623, 2006

[7] Massaro et al., Astroph.J., 376, L11-L15, 1991

[8] Ulmer et al., Astroph.J., 551, 244-248, 2001 